\title{
Serum Expression and Clinical Significance of Vascular Cell Adhesion Molecule-1 and MicroRNA-21 in Obstructive Sleep Apnea-Hypopnea Syndrome Patients in Chinese Population
}

\author{
DANDAN WU, AND BINGHUA XIA* \\ Department of Otolaryngology, Suzhou Ninth People's Hospital, Suzhou, Jiangsu 215000, China
}

Wu et al.: Serum expression and clinical significance of VCAM-1 and miR-21 in OSAHS patients

\begin{abstract}
The aim of this study was to detect the expression level of vascular cell adhesion molecule-1 and MicroRNA-21 in peripheral serum of adult Obstructive Sleep Apnea-Hypopnea Syndrome patients and healthy subjects, analyze the correlation between vascular cell adhesion molecule-1/microRNA-21 and the main indicators of polysomnography (apnea-hypopnea index, L-Saturation of Peripheral Oxygen) of subjects. To explore the relationship between the expression of vascular cell adhesion molecule-1/microRNA-21 and the severity of Obstructive Sleep Apnea-Hypopnea Syndrome and its clinical significance. Total of week 50 Obstructive Sleep Apnea-Hypopnea Syndrome patients and 27 control cases were collected as study objects from December 2018 to December 2019 in our hospital. Enzyme-linked immunoassay was used to detect the vascular cell adhesion molecule-1 expression. Quantitative Reverse Transcription-polymerase chain reaction analysis was performed to determine the MicroRNA-21 expression. The weight, body mass index and apnea-hypopnea index were higher while L-Saturation of Peripheral Oxygen was lower in both Mild and the Moderate-severe group Obstructive Sleep Apnea-Hypopnea Syndrome patients than control cases. Besides, the serum expression of vascular cell adhesion molecule-1 and MicroRNA-21 were increased significantly in Obstructive Sleep Apnea-Hypopnea Syndrome patients, and the level was positively correlated with the degree of the disease. We also demonstrated that vascular cell adhesion molecule-1 and MicroRNA-21 had a positive correlation with weight, body mass index and apnea-hypopnea index, whereas a negative correlation with L-Saturation of Peripheral Oxygen. Finally, there is a significant positive correlation between vascular cell adhesion molecule-1 and MicroRNA-21. We have proved that the expression level of vascular cell adhesion molecule-1 and MicroRNA-21 in serum of subjects is closely related to apnea-hypopnea index and L-Saturation of Peripheral Oxygen, which can reflect the severity of Obstructive Sleep Apnea-Hypopnea Syndrome and may become a new biological observation index for diagnosis and prognosis evaluation of Obstructive Sleep Apnea-Hypopnea Syndrome.
\end{abstract}

Key words: Obstructive sleep apnoea hypopnoea syndrome, Vascular cell adhesion protein -1, miR-21, Apnea Hypopnea Index, peripheral capillary oxygen saturation

Obstructive sleep apnea hypopnea syndrome (OSAHS) is a common and frequently occurring disease in otorhinolaryngology filed, mainly prevalent in men and its incidence rate increases with age $\mathrm{e}^{[1]}$. In addition, OSAHS patients have significantly increased the probability of cardiovascular and cerebrovascular diseases, which seriously affect the health and quality of life of patients, has attracted extensive attention ${ }^{[2,3]}$.

At present, there are many causes of OSAHS, which may be related to age, gender, obesity, chronic inflammation, hypoxia and heredity ${ }^{[4]}$. Polysomnography (PSG) monitoring, as the gold standard for diagnosis of OSAHS in clinic, can monitor many indexes, including sleep state and hypoxia degree of patients during sleep, the times of apnea and/or hypoventilation, sleep structure composition, degree and frequency of hypoxia during sleep at night, and distinguish the severity of OSAHS patients ${ }^{[5]}$. However, PSG monitoring also has some defects, such as high technical requirements, high cost and so on. Therefore, to study a simple, cheap and

*Address for correspondence

E-mail: shantoufu4145@163.com 
easy to promote and apply diagnostic method, such as: to find the biological markers related to OSAHS, will play a positive and beneficial role in the general survey and treatment of OSAHS patients.

Vascular cell adhesion molecule-1 (VCAM-1) is a kind of cell adhesion molecule, which can mediate the migration and extravasation of inflammatory cells from the junction of endothelial cells and play an important role in the process of chronic inflammatory diseases ${ }^{[6,7]}$. In recent $y$, studies have shown that the expression of VCAM-1 is significantly increased in patients with OSAHS, which may be involved in the development of OSAHS ${ }^{[8]}$. Additionally, MicroRNA-21 (miR-21) is an endogenous small nucleotide fragment, which plays an extremely important role in many biological processes such as cell proliferation, differentiation and apoptosis $^{[9]}$. Numerous of studies have shown that the expression level of miR-21 increases in a variety of hypoxic conditions or hypoxic diseases ${ }^{[10]}$. In addition, recent studies suggest that miR-21 expression in OSAHS is significantly upregulated ${ }^{[11]}$.

Given that VCAM-1 and miR-21 involved in a variety of anoxic and inflammatory diseases, whether they also play a role in the occurrence and development of OSAHS needed to be verified. In this study, VCAM1 and miR-21 were detected in peripheral serum of OSAHS patients with different severity, and the relationship between the two bio marks and severity of disease was observed, in order to explore novel serum molecular markers for severity evaluation of OSAHS patients.

\section{MATERIALS AND METHODS}

\section{Patient's collection:}

Total of 50 OSAHS patients who were diagnosed in Suzhou Ninth People's Hospital from December 2018 to December 2019 were collected as study objects, including 24 patients with mild and 26 patients with moderate-severe. Meanwhile, 27 healthy cases with no OSAHS were collected as the control group. The study was approved by the ethics committee of Suzhou Ninth People's Hospital. All patients agreed and signed the informed consent of clinical peripheral blood collection.

\section{Inclusion and exclusion criteria:}

Inclusion criteria: The patient's age is between 18 and 55 y old; the subjects with sleep snoring, suffocation and other clinical symptoms, who were diagnosed as OSAHS by PSG monitoring; the severity grading standard of OSAHS patients was determined according to the OSAHS diagnosis and treatment guidelines formulated by the sleep and respiratory disorders group of the Chinese Medical Association; the control group included in the experiment were excluded from OSAH by PSG monitoring; all subjects had no previous use history of special drugs, no history of acute or chronic upper respiratory tract infection or systemic fever within one $\mathrm{w}$ before PSG monitoring, and no smoking or alcohol for one w or more. Exclusion criteria: All subjects excluded sleep apnea caused by congenital stenosis of upper airway or acquired tumor formation. Subjects were excluded from the basic diseases that may interfere with the study of serum markers, such as hypertension, chronic lung disease, heart and brain infarction, diabetes, hyperthyroidism, grade 4 cardiac function or liver and kidney dysfunction, etc.; Those who suffered huge body or mental trauma in the near future; Those who drank coffee, strong tea and other drinks and took sedative and sleeping drugs within $12 \mathrm{~h}$ before PSG monitoring drug users.

\section{Enzyme-linked immunoassay (ELISA):}

Serum samples from all participants were collected and divided into different groups, and total serum VCAM1 levels were determined by ELISA kits (Bioscience, CA, USA). Detailed steps as previously described ${ }^{[12]}$. Finally, the Optical Density (OD) value of each reaction pore was measured by the enzyme scale instrument with the wavelength of $450 \mathrm{~nm}$, and the statistical analysis was carried out.

\section{Reverse transcription-quantitative real-time polymerase chain reaction (RT-PCR) analysis:}

Total RNA of serum samples subjects were extracted using Trizol according to the manufacturer's instructions. Reverse transcription of RNA was performed via a PrimeScript RT Kit (Bio-RAd,Shanghai), and qRT-PCR was using SYBR Premix kit (Bio-RAd,Shanghai). The results were normalized to the levels of U6 and then analysed using the $2^{-\triangle \Delta \mathrm{CT}}$ method. The primers used in the study were shown as below: miR-21, Forward Primer: 3'-TAACCGGGCCGACGTG-5'; Reverse Primer: 3'-TGGCAAAGCGCGAGT-5'; U6, Forward Primer: 3'-GTTGGCCGCCATAGG-5'; Reverse Primer: 3'-CTGGCACCTTCACCGG-5'.

\section{Statistical analysis:}

All data were presented as means \pm standard deviation (SD) using SPSS 22.0. Values were examined via the Student's t test for two groups, and one-way analysis 
of variance (ANOVA) with Tukey's post hoc test for three or more groups. $p$ value $<0.05$ was considered as statistically significant.

\section{RESULTS AND DISCUSSION}

There were no differences in age and gender among the Control group, the Mild group and the Moderate-severe group. However, the weight, body mass index (BMI) and apnea-hypopnea index (AHI) of the Mild group and the Moderate-severe group were both higher than those of the Control group. On the contrary, the L-Saturation of Peripheral Oxygen ( $\left(\mathrm{L}_{-} \mathrm{SPO}_{2}\right)$ of the Mild group and the Moderate-severe group were smaller than that of the Control group (Table 1). Besides, the serum expression of VCAM-1 and miR-21 were increased significantly in OSAHS patients, and the level was positively correlated with the degree of the disease, as shown in (fig. 1)

The serum expression of VCAM-1 in the Control group, the Mild group and the Moderate-severe group; The serum expression of miR-21 in the Control group, the Mild group and the Moderate-severe group. *meant $\mathrm{p}<0.05$ vs the Control group; ${ }^{*}$ meant $\mathrm{p}<0.05$ vs the Mild group.

Person correlation analysis was used to analysis the correlation between serum VCAM-1 and other variables. The results demonstrated that VCAM-1 had a positive correlation with weight, BMI, AHI and miR-
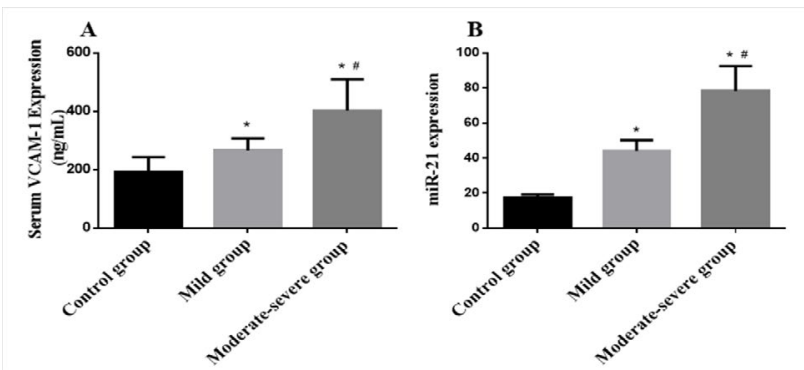

Fig. 1: The serum expression ofVCAM-1 and miR-21in different groups

TABLE 1: COMPARISON OF GENERAL INFORMATION AMONG THE THREE GROUPS

\begin{tabular}{|c|c|c|c|c|}
\hline Variables & $\begin{array}{l}\text { Control group } \\
(n=27)\end{array}$ & $\begin{array}{l}\text { Mild group } \\
(\mathrm{n}=24)\end{array}$ & $\begin{array}{l}\text { Moderate-severe group } \\
\qquad(\mathrm{n}=26)\end{array}$ & $p$ value \\
\hline Age $(y)$ & $39.23 \pm 9.34$ & $38.63 \pm 10.53$ & $39.75 \pm 11.21$ & 0.275 \\
\hline Gender & & & & 0.770 \\
\hline Male & 22 & 20 & 23 & \\
\hline Female & 5 & 4 & 3 & \\
\hline Weight (kg) & $58.84 \pm 8.99$ & $67.43 \pm 9.34^{*}$ & $74.11 \pm 10.18^{* \#}$ & $<0.001$ \\
\hline BMI $\left(\mathrm{kg} / \mathrm{m}^{2}\right)$ & $23.31 \pm 1.93$ & $25.82 \pm 2.01^{*}$ & $28.01 \pm 2.78^{\star \#}$ & $<0.001$ \\
\hline $\mathrm{AHI}$ & $2.43 \pm 1.28$ & $10.74 \pm 2.94^{*}$ & $37.28 \pm 12.45^{* \#}$ & $<0.001$ \\
\hline $\mathrm{L}-\mathrm{SPO}_{2}(\%)$ & $90.23 \pm 3.42$ & $82.99 \pm 4.20^{*}$ & $72.97 \pm 7.82^{* \#}$ & $<0.001$ \\
\hline
\end{tabular}

21 and a negative correlation with $\mathrm{L}-\mathrm{SPO}_{2}$. However, there was no significant correlation between VCAM-1 and age, as shown in (Table 2).

Person correlation analysis was used to analysis the correlation between serum miR-21 and other variables. The results showed that miR-21 had a positive correlation with weight, BMI, AHI and VCAM-1 and a negative correlation with $\mathrm{L}-\mathrm{SPO}_{2}$. However, there was no significant correlation between miR-21 and age, as shown in (Table 3).

For further study, we take AHI as the dependent variable, and take age, weight, BMI, L-SPO ${ }_{2}$, VCAM-1 and miR21 as the independent variables for multiple stepwise regression analysis. Finally, four variables including VCAM-1, miR-21, weight and L-SPO 2 were entered into the model, and the regression equation was AHI $=57.345+0.067 \mathrm{VCAM}-1+0.472 \mathrm{miR}-21+0.225$ weight$0.993 \mathrm{~L}-\mathrm{SPO}_{2}$, as shown in (Table 4).

The results showed that after treatment, the serum VCAM-1 and miR-21 expression and AHI were decreased significantly than those before treatment no matter in the Mild group or the Moderate-severe group, as shown in (fig. 2).

The AHI was decreased in OSAHS patients after treatment; the serum VCAM-1 expression was decreased in OSAHS patients after treatment; the serum miR-21 expression was decreased in OSAHS patients after treatment. *meant $\mathrm{p}<0.05$ vs before treatment.

Among the risk factors of many diseases, obesity plays an important role, the mechanism of the interaction between obesity and OSAHS has not been fully clarified $^{[13]}$. Narrowing of the upper airway, at multiple levels, is responsible for the consequences of OSAHS. Increased muscle tone in obese patients prevents the upper airways from collapsing during wake and the reverse occurs during sleep. Many studies showed that the accumulation of fat in neck and chest and abdomen

"meant $p<0.05$ vs the Control group; "meant $p<0.05$ vs the Mild group 
TABLE 2: CORRELATION ANALYSIS OF VCAM-1 AND OTHER VARIABLES

\begin{tabular}{lcccccc}
\hline Variables & Age & Weight & BMI & AHI & L-SP $_{0} 2$ & miR-21 $^{2}$ \\
\hline $\mathrm{r}$ & 0.089 & 0.523 & 0.454 & 0.873 & -0.782 & 0.725 \\
$\mathrm{p}$ value & 0.523 & $<0.001$ & $<0.001$ & $<0.001$ & $<0.001$ & $<0.001$ \\
\hline
\end{tabular}

TABLE 3: CORRELATION ANALYSIS OF MIR-21 AND OTHER VARIABLES

\begin{tabular}{lcccccc}
\hline Variables & Age & Weight & BMI & AHI & L-SPO $_{2}$ & VCAM-1 \\
\hline $\mathrm{r}$ & 0.128 & 0.492 & 0.563 & 0.734 & -0.822 & 0.725 \\
$\mathrm{p}$ value & 0.923 & $<0.01$ & $<0.01$ & $<0.001$ & $<0.001$ & $<0.001$ \\
\hline
\end{tabular}

TABLE 4: MULTIPLE STEPWISE REGRESSION ANALYSIS OF INFLUENCING FACTORS OF AHI

\begin{tabular}{lccccc}
\hline Variables & $\begin{array}{c}\text { Partial regression } \\
\text { coefficients }\end{array}$ & Standard error & $\begin{array}{c}\text { Standard partial regression } \\
\text { coefficient }\end{array}$ & $\mathbf{t}$ & $\mathrm{p}$ \\
\hline VCAM-1 & 0.067 & 0.011 & 0.040 & 6.324 & $<0.001$ \\
miR-21 & 0.472 & 0.093 & 0.273 & 4.728 & $<0.001$ \\
Weight (kg) & 0.225 & 0.073 & 0.147 & 3.284 & 0.001 \\
$\mathrm{~L}-S P O_{2}(\%)$ & -0.993 & 0.133 & -0.472 & -6.579 & $<0.001$ \\
Constant & 57.345 & 16.732 & - & 3.782 & 0.001 \\
\hline
\end{tabular}
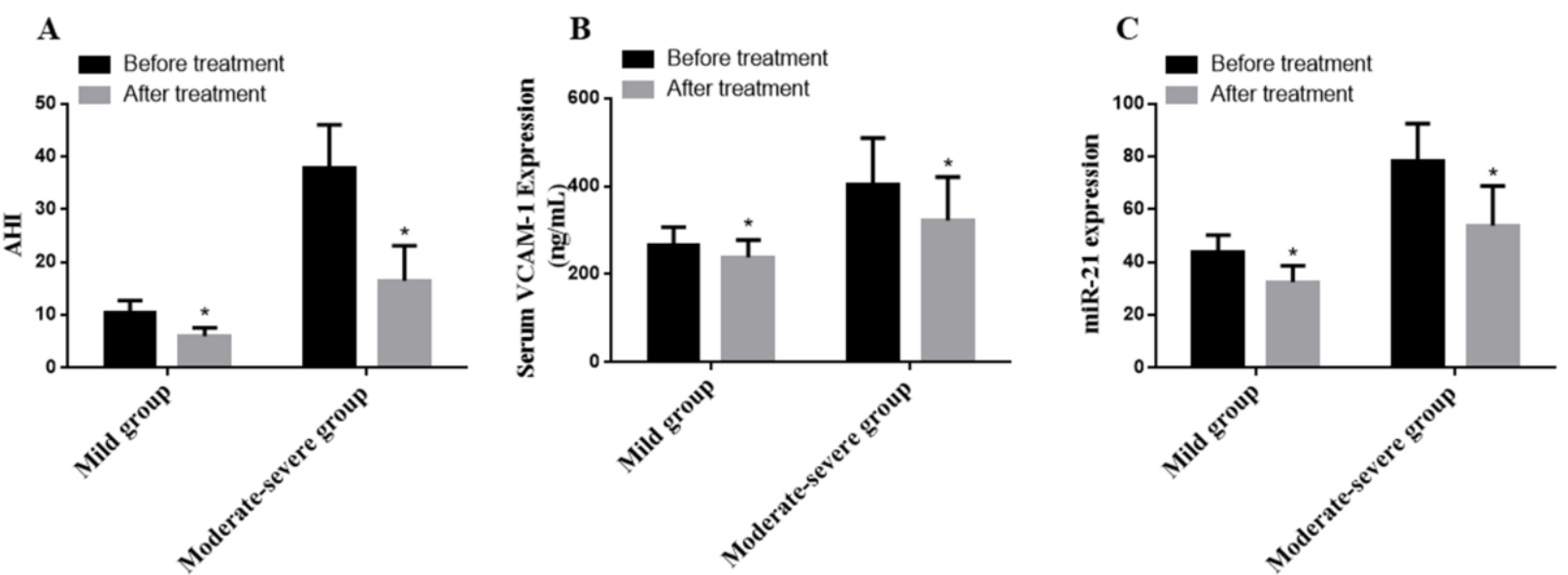

Fig. 2: The serum expression of AHI, VCAM-1 and miR-21in different groups after treatment

of obese patients can increase airway resistance, which is the main cause of airway obstruction ${ }^{[14]}$. In our study, we also found that BMI and AHI were significantly higher, while $\mathrm{L}-\mathrm{SPO}_{2}$ was lower in OSAHS patients than that of control cases.

VCAM-1, as an important cell adhesion molecule, is widely expressed on the surface of activated endothelial cells, smooth muscle cells, macrophages and so on ${ }^{[15]}$. The most important role of VCAM-1 in vascular endothelial cells is that it can induce adhesion of leukocytes and inflammatory factors to promote vascular endothelial inflammation ${ }^{[16,17]}$. In the process of OSAHS, hypoxia/reoxygenation can promote the overexpression of inflammatory related susceptible genes, which can increase the synthesis of inflammatory factors, and then lead to chronic inflammatory response and multi system and multi organ damage ${ }^{[18,19]}$. It has also been reported that VCAM-1 is significantly increased in OSAHS patients ${ }^{[20]}$. In our study, we demonstrated that VCAM-1 had a positive correlation with weight, BMI, AHI and miR-21 and a negative correlation with $\mathrm{L}-\mathrm{SPO}_{2}$.

The understanding and study of miR-21 are mainly focused on some malignant tumors, such as gastric cancer, colorectal cancer, breast cancer, lung cancer, etc $^{[21]}$. Currently, it has been found that miR-21 can also mediate inflammation and oxidation of the body ${ }^{[22]}$. By upregulating the expression of miRNA-21, it can inhibit inflammation and apoptosis of renal tubular epithelial cells, and improve the acute renal injury induced by ischemia/reperfusion $^{[12]}$. In this study, qRT-PCR was used to detect the content of miR-21 in sera of OSAHS patients with different degrees of disease and healthy subjects. Meanwhile, the correlation between miR-21 and AHI, L-SPO ${ }_{2}$, which reflect the severity of disease, was analyzed. It was found that miR-21 was positively correlated with AHI and negatively correlated with L-SPO . 
In conclusion, we found that the expression levels of VCAM-1 and miRNA-21 in the peripheral serum of the subjects increased with the aggravation of OSAHS, and were significantly correlated with the important indicators of polysomnography (AHI, L-SPO ${ }_{2}$ ). In addition, there is a significant positive correlation between VCAM-1 and miRNA-21, indicating that both of them participate in the disease process of OSAHS, and can reflect the severity of the disease, which is expected to become a suitable biomarker of OSAHS in the future.

\section{Authors' contributions:}

Binghua Xia conceived and designed the experiments; Dandan $\mathrm{Wu}$ analyzed the data and wrote the paper.

\section{Acknowledgement:}

This work was supported by Suzhou Ninth People's Hospital.

\section{Conflict of Interests:}

The authors declared no conflict of interest.

\section{REFERENCES}

1. Tetter N, Tschopp K. Contribution of hyoid and tonsillar procedures to outcome in multilevel surgery for obstructive sleep apnea syndrome. OtorhinolaryngolRelat Spec 2016;78:353-60.

2. Zeng Y, Yang S, Wang X, Fan J, Nie S, Wei Y. Prognostic impact of residual syntax score in patients with obstructive sleep apnea and acute coronary syndrome: A prospective cohort study. Respir Res 2019;20:43.

3. Macey PM, Kumar R, Ogren JA, Woo MA, Harper RM. Global brain blood-oxygen level responses to autonomic challenges in obstructive sleep apnea. PLoS One 2014;9:e105261.

4. Barnes H, Edwards BA, Joosten SA, Naughton MT, Hamilton GS, Dabscheck E. Positional modification techniques for supine obstructive sleep apnea: A systematic review and metaanalysis. Sleep Med Rev 2017;36:107-15.

5. Nerfeldt P, Sundelin A. Obstructive sleep apnea in children with Down syndrome-prevalence and evaluation of surgical treatment. Int J PediatrOtorhinolaryngol 2020;133:109968.

6. Wu JZ, Liu YH, Liang JL, Huang QH, Dou YX, Nie J, et al. Protective role of beta-patchoulene from pogostemoncablin against indomethacin-induced gastric ulcer in rats: Involvement of anti-inflammation and angiogenesis. Phytomedicine 2018;39:111-8.

7. Chen Y, Yang W, Xu C. Expression of vascular cell adhesion molecule 1 (VCAM-1) in the mammary lymph nodes of cows with subclinical mastitis. J Vet Res 2017;61:203-9.

8. Wang J, Li X, Hou WJ, Dong LX, Cao J. Endothelial function and T-lymphocyte subsets in patients with overlap syndrome of chronic obstructive pulmonary disease and obstructive sleep apnea. Chin Med J 2019;132:1654-9.

9. Dioguardi M, Caloro GA, Laino L, Alovisi M, Sovereto D, Crincoli V, et al. Circulating mir-21 as a potential biomarker for the diagnosis of oral cancer: A systematic review with meta-analysis. Cancers 2020;12:936.

10. Li C, Fei K, Tian F, Gao C, Song Y. Adipose-derived mesenchymal stem cells attenuate ischemic brain injuries in rats by modulating mir-21-3p/mat $2 \mathrm{~b}$ signaling transduction. Croat Med J 2019;60:439-48.

11. Chen YC, Hsu PY, Su MC, Chin CH, Liou CW, Wang $\mathrm{TY}$, et al. miR-21-5p under-expression in patients with obstructive sleep apnea modulates intermittent hypoxia with re-oxygenation-induced-cell apoptosis and cytotoxicity by targeting pro-inflammatory TNF- $\alpha$-TLR4 signaling. Int $\mathrm{J}$ MolSci 2020;21:999.

12. Zhang W, Shu L. Upregulation of miR-21 by ghrelin ameliorates ischemia/reperfusion-induced acute kidney injury by inhibiting inflammation and cell apoptosis. DNA Cell Biol 2016;35:417-25.

13. Ghazal A, Roghani F, Sadeghi M, Amra B, KermaniAlghoraishi M. Obstructive sleep apnea, diagnosed by the berlin questionnaire and association with coronary artery disease severity. ARYA Atheroscler 2015;11:275-80.

14. $\mathrm{Xu} X, \mathrm{Xu}$ J. Effects of different obesity-related adipokines on the occurrence of obstructive sleep apnea. Endocr J 2020;67:485-500.

15. Hocaoglu-Emre FS, Saribal D, Yenmis G, Guvenen G. Vascular cell adhesion molecule 1, intercellular adhesion molecule 1, and cluster of differentiation 146 levels in patients with Type 2 diabetes with complications. EndocrinolMetab 2017;32:99105.

16. Carter RA, Wicks IP. Vascular cell adhesion molecule 1 (CD106): A multifaceted regulator of joint inflammation. Arthritis Rheum 2001;44:985-94.

17. OrndorffRL, Hong N, Yu K, Feinstein SI, Zern BJ, Fisher $\mathrm{AB}$, et al.NOX2 in lung inflammation: Quantum dot based in situ imaging of NOX2-mediated expression of vascular cell adhesion molecule-1. Am J Physiol Lung Cell MolPhysiol 2014;306:L260-8.

18. Godoy J, Mellado P, Tapia J, Santin J. Obstructive sleep apnea as an independent stroke risk factor: Possible mechanisms. CurrMolMed 2009;9:203-9.

19. Farre R, Montserrat JM, Gozal D, Almendros I, Navajas D. Intermittent hypoxia severity in animal models of sleep apnea. Front Physiol 2018;9:1556.

20. Kaczmarek E, Bakker JP, Clarke DN, Csizmadia E, Kocher O, Veves A, et al. Molecular biomarkers of vascular dysfunction in obstructive sleep apnea. PLoS One 2013;8:e70559.

21. Bautista-Sanchez D, Arriaga-Canon C, Pedroza-Torres A, De La Rosa-Velazquez IA, Gonzalez-Barrios R, ContrerasEspinosa L, et al. The promising role of miR-21 as a cancer biomarker and its importance in RNA-based therapeutics. MolTher Nucleic Acids 2020;20:409-20.

22. Darabi F, Aghaei M, Movahedian A, Elahifar A, Pourmoghadas A, Sarrafzadegan N. Association of serum microrna-21 levels with visfatin, inflammation, and acute coronary syndromes. Heart Vessels 2017;32:549-57.

This is an open access article distributed under the terms of the Creative Commons Attribution-NonCommercial-ShareAlike 3.0 License, which allows others to remix, tweak, and build upon the work non-commercially, as long as the author is credited and the new creations are licensed under the identical terms

This article was originally published in a special issue,
"Biomedical Research in Clinical and Preclinical
Pharmaceutics" Indian J Pharm Sci 2020:82(3)Spl issue7;73-77

